\title{
Structural analysis and dynamic Young's modulus measurement of selected tropical wood polymer composites
}

\begin{abstract}
Various tropical wood species are abundantly available in Southeast Asia, especially in Borneo Island. In this study, selected tropical wood species, Eugenia spp., Artocarpus rigidus, Artocarpus elasticus, Koompassia malaccensis and Xylopia spp., were chemically treated with sodium meta periodate to convert them into wood polymer composites (WPCs). Manufactured WPCs were characterised using microstructural analysis (Fourier transform infrared spectroscopy and SEM) and dynamic Young's modulus measurement. The dynamic Young's modulus was calculated using vibrational testing $\mathrm{E} d$ and dynamic mechanical thermal analysis E'. Spectroscopic analysis results indicated the absorption band of raw wood at $1635 \mathrm{~cm}-1$ due to carbonyl stretching and WPCs showed increased absorption band near $1718 \mathrm{~cm}-1$. The manufactured WPCs yielded higher Young's modulus compared with raw woods. A. elasticus WPC had the highest Young's modulus compared with the other composites.
\end{abstract}

Keyword: Structural analysis; Dynamic Youngs modulus; Tropical wood polymer composites. 